\title{
Primitive Ontology and the Classical World
}

\author{
Valia Allori \\ Philosophy Department, Northern Illinois University
}

Forthcoming in: R. Kastner, J. Jeknic-Dugic, G. Jaroszkiewicz (eds.), Quantum Structural Studies: Classical Emergence from the Quantum Level. World Scientific Publishing Co.

\begin{abstract}
In this paper I present the common structure of quantum theories with a primitive ontology, and discuss in what sense the classical world emerges from quantum theories as understood in this framework. In addition, I argue that the primitive ontology approach is better at answering this question than the rival wave function ontology approach or any other approach in which the classical world is nonreductively 'emergent:' even if the classical limit within this framework needs to be fully developed, the difficulties are technical rather than conceptual, while this is not true for the alternatives.
\end{abstract}

\section{Introduction}

Scientific realists believe that, in some strong sense, physical reality is accessible to us through scientific investigation. In particular, scientists can account for what exist by looking at our best scientific theories. This view has been challenged by the success of quantum mechanics: while, on the one hand, quantum theory is incredibly powerful in making new and very precise predictions, on the other hand it is extremely difficult to understand what image of the world this theory provides us. Indeed, many have thought that the real lesson of quantum mechanics is that the dream of the scientific realist is impossible, since quantum mechanics has been taken to suggest that physical objects have contradictory properties, like being in a place and not being in that place at the same time, or that properties do not exist at all independently of observation ${ }^{1}$. Naturally, scientific realists accepted the challenge of 'making sense' of the quantum world, and today we have various proposal of quantum theories that allow for a realist reading. Among these theories, most famously we find Bohmian and Everettian mechanics, and the GRW theory: they are fundamental physical theories according to which there is an objective physical world, which can be described by noncontradictory, mind-independent properties. Most philosophers of physics recognize the legitimacy of these theories, but disagree about the metaphysical pictures these theories actually provide. Many scientific realists think that the wave function represents material objects: they defend a view that have been dubbed 'wave function ontology.' Others instead believe that it is not the case. The primitive ontology (PO) approach belongs to the latter group: the idea is that in all quantum theories (and more

${ }^{1}$ The claim that Bohr himself was a positivist has been challenged by [Bai \& Stachel, this volume]. Nonetheless, if not Bohr, many others have turned to positivism because of these considerations. 
in general, in all fundamental physical theories), the microscopic description of reality is provided by an object which, mathematically, is defined in three-dimensional space (or four-dimensional space-time), which has been dubbed the primitive ontology of the theory. As we will wee, the wave function, being a different kind of mathematical entity, is not a suitable PO for quantum theories. Rather, it has a different role in these theories: instead of describing microscopic reality, it is a necessary ingredient in defining the law of motion for the microscopic constituents of the world described by the PO.

In this paper, I wish to analyze in more detail than previously discussed in the literature the connection between the microscopic description of reality provided by quantum theories in the $\mathrm{PO}$ framework and the macroscopic, classical world of our everyday experience. In the next two sections, I describe the PO approach, first outlining the main ideas and then describing quantum theories with a PO. Then, in section 4, I discuss the 'general scheme of mechanics,' namely the main ingredients and the fundamental structure that fundamental physical theories with a PO have in common and which is used to account for the behavior of matter according to the theory. In section 5, I analyze the classical limit of quantum theories in the PO framework. Then I draw a contrast with the wave function ontology approach. Given that the wave function does not live in three-dimensional space, there is the problem of accounting for our three-dimensional perception of the macroscopic world. People defending the PO approach believe that this gap between the manifest and the scientific image is very hard to fill without too high a cost: quantum theories understood in these terms will cease to be simple and will lose explanatory power. The primitive ontologists instead maintain that, when considering quantum theories with a PO, then one can construct a simple and suitably straightforward explanatory schema which can be used to explain everyday macroscopic phenomena in terms in quantum microscopic ones. As we will see, this schema, in contrast to what happens in the wave function ontology framework, is an extension of the one used to account for macroscopic phenomena in terms of the microscopic entities in classical physics. In this way, quantum theories understood as theories with a primitive ontology are simpler and more explanatory.

\section{The Main Ideas of the Primitive Ontology Approach}

By looking to the history of science, it seems that mathematics can describe the world around us. This is a remarkable and impressive fact, which I will not attempt to account for in this paper. Nonetheless, if nature speaks in the language of mathematics and our aim is to study and explain the world around us, our theory will be a mathematical theory of which we will give a physical interpretation. But what does this really mean? In this paper, I will discuss the answer provided by the PO approach, and in this section 
I will summarize its main ingredients ${ }^{2}$. Ultimately, scientific realists want fundamental physical theories to explain and account for the objects of their experience, which are given directly to them. Thus, the theory will need to specify which mathematical objects, among the variety it contains, should be taken to represent physical objects. Once this has been established, the other mathematical entities in the theory will find their place accordingly. Concisely, the basic idea of the PO framework rests on the recognition that in a fundamental physical theory, some mathematics is representational and some is not. Some mathematical entities in the theory will represent matter (otherwise the theory will be just devoid of physical significance): these entities are the PO of the theory. Some other mathematical objects will have the role of determining how matter moves in time: these are the so-called 'nomological' variables of the theory. And some other mathematical objects represent nothing in the world: they are just useful tools necessary to formalize the theory as it is. Other (realist) approaches share this idea, but the $\mathrm{PO}$ approach is peculiar in the fact that a suitable $\mathrm{PO}$ has to meet the following characteristics: (1) it needs to be defined in three-dimensional space (or four-dimensional space-time) rather than being a more complicated mathematical object; and (2) it needs to be microscopic rather than macroscopic. Roughly, the three-dimensionality of the primitive variables allows for a direct contact between the variables in the theory and the objects in the world we want them to describe. In fact, a PO represented by an object in a space of dimension $d$, different from three, would imply that matter lives in a $d$-dimensional space. Thus, our fundamental physical theory would have to be able to provide an additional explanation of why we think we live in three-dimensional world while we actually do not. It has been argued that this is at best undesirable, in part for reasons connected to another feature that a good $\mathrm{PO}$ is supposed to have, namely its fundamentality ${ }^{3}$. Also, the primitive variables better be microscopic entities: a microscopic $\mathrm{PO}$, in which the PO constitutes the building blocks of everything else, is able to ground a (bottom-up) scheme of explanation that allows determining the properties of macroscopic physical objects in terms of the behavior of the PO, as we will explain later in this paper.

In order to provide a simple example of the $\mathrm{PO}$ approach that I will later use to explain how the classical world emerges from quantum theories, let us consider classical mechanics. According to this theory, matter is made of point particles. These particles are completely described by their position $r$, which is represented by coordinates in three-dimensional Euclidean space, taken to represent physical space. Particles' positions are the $\mathrm{PO}$ of the theory. The particles move in time according to a

${ }^{2}$ For a more detailed discussion of this approach, see e.g. [AGTZ 2008], [Allori 2013a; 2013b; 2015a; forthcoming] and references therein.

${ }^{3}$ In this regard, see [Allori 2013a; 2013b] and [Monton 2006].

${ }^{4}$ See footnote 14 for an example of a theory with a macroscopic PO, and for an argument for why this is at best unsatisfactory. 
definite law of motion, given by Newton's second law. In order to specify this law, $=$ $m \frac{d^{2} r}{d t^{2}}$, additional mathematical objects are required: $m$ specifies the mass of the particle, and $F$ is the external force that produces in the body the acceleration $a=\frac{d^{2} r}{d t^{2}}$. In addition, one needs to specify the so-called laws of the force, namely what $F$ looks like. In the case of Newton's theory of gravitation, we have the familiar $F=G \frac{m_{1} m_{2}}{r_{12}}$, where $G$ is the gravitational constant, $m_{i}, i=1,2$ represent the masses of the bodies involved, and $r_{12}$ is their relative spatial distance. Nothing changes much in this respect if we move to classical electrodynamics: the only difference will be that one would have to specify the suitable expressions for the electromagnetic force, namely $F=q_{1} v \times B+\frac{1}{4 \pi \varepsilon_{0}} \frac{q_{1} q_{2}}{r_{12}}$, which includes the constant $\varepsilon_{0}$, the particle velocity $v$, the charges $q_{i}, i=1,2$, and the magnetic field $B$. In contrast with the PO, these variables do not 'make stuff up:' matter is not made of forces, or of masses. Matter is made by particles which are subject to forces in virtue of their masses. Nonetheless, these variables are needed to account for 'how stuff moves.' This is where the name 'nomological variables' comes from: they help define the law of nature with which matter evolves ${ }^{5}$.

\section{Quantum Theories with Primitive Ontology}

Here is how the PO approach extends to the quantum framework. Let us focus on some quantum theories which are compatible with scientific realism, namely Bohmian mechanics [Bohm 1952], the GRW theory [GRW 1986], and Everettian mechanics (also known as the Many-Worlds theory) [Everett 1957]. As we will see in the following section, they have the following structure in common: matter is described by the PO (which is particles, matter field, or flashes); the temporal evolution of matter is determined by the wave function, which is the main nomological variable. The wave function has its own evolution equation: it can evolve linearly (according to the 'usual' Schrödinger equation), or stochastically (according to the GRW evolution discussed later).

These theories emerged as a response to the infamous measurement problem, which plagued early quantum theories and lead many people into embracing antirealism. If matter is completely described by the wave function, and it evolves according the Schrödinger equation, then impossible macroscopic superpositions, like a dead and alive cat, promptly arise. To avoid such superpositions, many have thought

5 Does the electromagnetic field belong to the PO? On the one hand, one would say that it does, for it is both three-dimensional and microscopic: in this sense, the electromagnetic field is a local beables [Bell 1987]. On the other hand, though, its role in the theory seems to be the one of generating the trajectories of matter rather than representing matter itself. If so, the electromagnetic field is a nomological variable (even if it is microscopic and three-dimensional). A discussion on the metaphysical status of the electromagnetic field can be found in [Allori 2015c]. 
that one either had to add something to the wave function, or have it evolve according to another equation [Bell 1987]. Bohmian mechanics is usually taken to be an example of the first kind of theories: there is the wave function, but in addition there are particles. Not so in the PO approach: the world is not made of wave function, rather only particles are the PO of Bohmian mechanics. In fact, the wave function is a mathematical object that lives in a very abstract space, namely configuration space: the space of all the positions of all the particles in the universe, configuration space. If there are $N$ particles in the universe, configuration space has dimension $M=3 N$. Thus by definition, the wave function is not a suitable primitive variable, given that it fails the first condition to be a PO. In addition, in Bohmain mechanics the trajectories of a system of particles are determined by Bohm's guidance equation, which involves the wave function that in turn evolves in time according to Schrödinger's equation ${ }^{6}$. Thus, the wave function has the role of generating the trajectories of the $\mathrm{PO}$ of Bohmian mechanics, and consequently should be regarded as a non-primitive variable ${ }^{7}$.

The situation in the GRW theory is more complicated: what is the PO of the GRW theory? Historically, the GRW theory was proposed as a solution of the measurement problem where the macroscopic superpositions were 'cured' by having the wave function collapse into one of the terms of the superpositions as a matter of law of nature. This is the reason why sometimes the theory is called 'spontaneous collapse theory.' The wave function first evolves according to the Schrödinger equation until a random time. At that time, the wave function undergoes an instantaneous collapse around a point which is chosen randomly according to a given, suitably defined, probability distribution in terms of the wave function. Then the situation repeats itself: the wave function continues to evolve according to the Schrödinger equation until a second random time, after which it collapses again around a second collapse center, et cetera, et cetera ${ }^{8}$. As such, GRW has been taken to be a theory in which matter is

\footnotetext{
${ }^{6}$ For a simple but mathematically accurate description of Bohm's theory, see [Allori \& Zanghi 2004].

${ }^{7}$ The PO approach takes three-dimensional space (or four-dimensional space-time) as fundamental, in the sense that it provides the arena in which physical phenomena take place, and as such the PO view is in contrast with others approaches in which space-time somehow 'emerges' from the basic nonspatiotemporal framework. Examples of such approaches go from the wave function ontology approach in which the fundamental space is configuration space, to the transactional interpretation discussed in [Kastner, this volume], which is based on "an intrinsically unobservable, pre-spacetime quantum substratum" from which space-time emerges. In addition we have, for instance, the system developed in [Baumgarten, this volume], in which space-time is emergent in a relationist sense, and fundamental physical theories are taken to "describe how entities change," rather than "what entities are." This seems to suggest some sort of structuralism, while the PO approach seems arguably closer to a limited realism $a$ la Kitcher [Kitcher 1993], even if recently it has been argued otherwise [Esfeld forthcoming]. These issues are worthwhile investigating, and a preliminary assessment can be found in [Allori manuscript].

${ }^{8}$ The reader interested in a mathematical description of the GRW evolution in terms of the wave function as discussed here is encouraged to read [AGTZ 2008].
} 
described by the wave function ${ }^{9}$, for the wave function is the only object whose temporal evolution was described in the theory as originally presented. As we have already discussed, though, in the PO approach the wave function cannot represent matter. Thus, as it stands, the GRW theory is fundamentally incomplete because it misses a suitable PO. One modification of the GRW theory to include a suitable PO has first been put forward in [BGG 1995] and later dubbed GRWm in [AGTZ 2008]. In this theory the PO is a three-dimensional matter field $m$ defined roughly as the sum of the masses of each 'particle,' weighed by the square module of the wave function. In this way, just as in Bohmian mechanics, the wave function has the role of implementing the law of temporal evolution for the $\mathrm{PO}$, for it defines the probability distribution that determines where the matter density will collapse. Another proposal of a GRW theory with a different PO was first suggested in [Bell 1987], then adopted in [Tumulka 2006] and called GRWf in [AGTZ 2008]. In this theory, the PO is represented by space-time events, dubbed 'flashes.' These flashes are randomly distributed in space-time in a way determined by the GRW-evolving wave function: every flash corresponds to one of the spontaneous collapses of the wave function. As in GRWm, the wave function has the role of generating the distribution of the flashes ${ }^{10}$. Another possibility is a theory dubbed GRWp3 in [AGTZ 2014]. This theory has a PO of particles, whose motion is governed by an equation that is exactly like Bohm's guiding equation. The difference is that in this theory the wave function obeys a GRW-like evolution such that the collapses occur exactly as in usual GRW theories except that, once the time for the collapse has been chosen, the collapse is centered at the actual position of the particle displaced at random. To sum up, in the PO framework, there is no single GRW theory: there are instead (at least) GRWm, GRWf and GRWp3. Each theory describes matter differently, respectively as made of a continuous material field, as made of flashes, and as made of particles. In contrast, the GRW-evolving wave function in each of these theory has the same role: it governs the motion of matter. In this framework, then, the fundamental difference between Bohmian mechanics and the GRW theory is not that the former solves the measurement problem supplementing the description provided by the Schrödinger evolving wave function with particles, while in the latter the wave function provides the complete description of the world but it does not evolve according to the Schrödinger equation. In fact in both theories the PO needs to be specified in addition to the wave function. Since the wave function does not represent matter, the fact that in Bohmian mechanics the wave function evolves according to the Schrödinger equation and in the GRW-like theories it does not, does not constitute a fundamental difference.

\footnotetext{
${ }^{9}$ See, for instance, [Albert 1996].

${ }^{10}$ [Chiatti and Licata, this volume] write that they "assume the only truly existent 'thing' existent in the physical world is events of creation and destruction." I believe that a question that is worth investigating is whether or not they have in mind a theory of flashes in which the spatiotemporal distribution of the flashes is determined by a Schrödinger evolving wave function.
} 
Indeed, one can formulate, as shown in [AGTZ 2008], the former in terms of a 'collapsed' wave function, and one of the latter, namely GRWf, in terms of a Schrödinger evolving one. The real difference is that while in Bohmian mechanics the PO is independent of the wave function, in GRW-like theories like GRWm, GRWf and GRWp3 the PO is defined in terms of it: $\mathrm{PO}=f(\psi)$. In this sense, the PO supervenes on the wave function, but naturally and not logically ${ }^{11}$.

Lastly, let us consider Everettian mechanics. It was originally proposed to solve the measurement problem by allowing for each macroscopic superpositions to be realized, each in a different world (hence the name 'many worlds' often used to dub the theory). In virtue of this, nothing is added to the Schrödinger-evolving wave function. Accordingly, almost all the proponents of Everettian mechanics agree in considering the wave function as the object in the theory that describes physical objects ${ }^{12}$. Again, this is at odds with the PO approach I am analyzing here. Nonetheless, a theory originally developed in [Bell 1987] can be regarded as an Everettian theory with a PO of particles. In this theory, the wave function evolves according to the Schrödinger equation and guides the motion of the particles, like in Bohmian mechanics. But here the particles do not have a continuous trajectory in space-time, rather, there is no temporal correlation among them. The theory was later dubbed BMW (that is, Bell Many Worlds) in [AGTZ 2008] and later called Sip (S from the Schrödinger evolution of the wave function, i for independent, $p$ for particle ontology) in [AGTZ 2011]. [AGTZ 2011] also describe another Everettian theory with a three-dimensional matter field PO, whose evolution is determined by a Schrödinger evolving wave function. This theory has been dubbed Sm ( $\mathrm{S}$ for the Schrödinger equation and $\mathrm{m}$ for the matter density function). It is also possible to construct a many-worlds like theory with a PO of flashes. An example of such a theory is Sf as described in [AGTZ 2011], in which a Schrödinger evolving wave function never collapses and determines the distribution of the flashes, hence the name.

All these theory have a many-worlds character, given the linearity of the Schrödinger evolution to which the wave function evolves. In fact, contrarily to Bohmian mechanics in which the positions of the particles are connected by the Bohmian trajectories, in Sip there is no connection whatsoever between where a particle is at a given instant of time and where it will be at the previous or following instant. As a consequence, many worlds exist, not at the same time, but one after another. In Sm, the superpositions of the wave function are inherited by the matter density field and therefore there are non-interacting mass densities associated with the different terms of the superposition. Thus, they can indeed be regarded as comprising many worlds,

\footnotetext{
${ }^{11}$ Roughly, $Y$ supervenes logically on $X$ when once God has created a world with certain $X$, the $Y$ comes along for free; instead $Y$ supervenes naturally on $X$ when after making the $X$, God had to do more work in order to make the $\mathrm{Y}$ : he had to make a law relating the $\mathrm{X}$ and the $\mathrm{Y}$. Once the law is defined, $\mathrm{X}$ will automatically bring along the $\mathrm{Y}$.

${ }^{12}$ See, e.g. [Wallace 2002].
} 
superimposed on a single space-time. Since the different worlds do not interact among themselves, they are, so to speak, reciprocally transparent. Similarly, in Sf different noninteracting families of flashes correspond to different terms of the superposition, and hence the many-worlds character of the theory.

In addition to the theories already described, we can imagine a variety of other theories mixing up the various types of $\mathrm{PO}$ and the various evolution equations, with the only additional constraint that they are empirically adequate ${ }^{13}$. To summarize, according to the $\mathrm{PO}$ approach, philosophers of physics and in general scholars interested in the foundations of quantum mechanics have always focused on the wave function, but that was a mistake: the wave function should not be taken as representing material objects. For if one does, she will have to face problems like the measurement problem, which arise due to the mathematical properties of the wave function, and which disappear on the $\mathrm{PO}$ approach. This framework says that in quantum theories, just like in any other fundamental physical theories, matter is represented by a variable in three-dimensional space (or four-dimensional space-time), and the wave function has the role of implementing the law of evolution for the PO. Thus, the wave function does not describe matter, it describes how matter behaves ${ }^{14}$.

\section{The General Scheme of Mechanics}

Let us get back to classical mechanics for a moment. As we saw above, schematically, Newtonian mechanics accounts the behavior of macroscopic objects as follow:

1) It specifies what material objects are made of (the PO);

2) It specifies how the PO moves in time (the law of motion for the PO);

3) In order to do this, it specifies the laws of the force.

Typically, one talks about the state of the system to specify the quantities that provide the complete dynamical description of physical bodies. In Newton's theory, the state is given by the pair $(r, p)$ of the position and momentum of the particles. As we mentioned already in Section 2, the position of particles represents the PO, and the momentum is the nomological variable: what is needed to implement the law of motion for the PO. In fact, in terms of these variables, one can rewrite Newton's second law as a

\footnotetext{
${ }^{13}$ For more on these theories, see [AGTZ 2008; 2011; 2014], and [Allori 2013b; 2015a; forthcoming].

${ }^{14}$ It is interesting to notice how quantum theories with a macroscopic PO seem to have been proposed. In fact, as first discussed in [AGTZ 2008], one could think of Bohr's quantum theory as one example of this: what might be regarded as its PO is the classical description of macroscopic objects which Bohr insisted was indispensable, with the wave function serving to determine the probability relations between the successive states of these objects. Since the notion of 'macroscopic' entities is hopelessly vague, the theory is not satisfactory. In addition, as also discussed in [Kastner, this volume], Bohr's quantum theory merely postulates rather than explains the emergence of the classical behavior from the quantum world, and because of this seems extremely ad hoc.
} 
set of two first-order differential equations, one for the evolution of position, and the other for the evolution of momentum as follows:

$$
\frac{d r}{d t}=u(r, p), \quad \frac{d p}{d t}=F(r, p),
$$

where $u=\frac{p}{m}$, where $m$ is the mass, and $F$ is the force. These equations are the general equations of mechanics: the first defines the velocity, and the second its rate of change. Together, via the specification of $u$ and $F$, they determine how the PO moves in time. If the PO approach is correct, then this general scheme is not a peculiarity of Newton's theory, but arguably a general trait of a fundamental physical theory ${ }^{15}$. The claim is that in general the state of the system is always given by the pair $(\chi, \eta)$, where $\chi$ represents the $\mathrm{PO}$, and $\eta$ the nomological variable. Typically, the PO's temporal evolution is defined in terms of some function $u$, and the nomological variable temporally evolves as specified by another function $F$.

That is:

$$
\text { temporal evolution of } \chi=u, \quad \text { temporal evolution of } \eta=F \text {. }
$$

In Newton's theory, as we saw, the $\mathrm{PO}$ is $\chi=r$, and the nomological variable is $\eta=p$. In addition, $u=\frac{d r}{d t}=p / m$ and $F=m \frac{d u}{d t}$, where $F=-\nabla V(r), V$ being the (gravitational) potential. The theory is defined when the spatiotemporal trajectories of the PO $\chi=\chi(t)$ are given, and this is determined once $u$ and $F$ are specified.

In the framework of quantum theories, if the PO evolves deterministically, the scheme described above for Newtonian mechanics is generalized rather straightforwardly. The temporal evolution of $\chi$ and $\eta$ can be described by two functions, $u$ and $F$, such that one can write an equation for the evolution of $\chi$ in terms of an appropriate function $u$ and one can write an equation for $\eta$ in terms of another function $F$. Consider, for instance, Bohmian mechanics. In this theory, the world is composed of particles, described by points in three-dimensional space, like in Newtonian mechanics. The pair $(r, \psi)$, where $\psi$ is the wave function and $r=\left(r_{1}, \ldots, r_{N}\right)$ represent the configuration of all the $N$ particles under consideration, are the state of a system. To stress the formal analogies with Newtonian mechanics, let us use the same notation we used before. We have: $\chi=r, \eta=\psi$. The functions $u$ and $F$ are defined as follows:

$$
u=\frac{\hbar}{m} \operatorname{Im}\left[\frac{\eta^{*} \nabla \eta}{\eta^{*} \eta}\right], \quad F=\frac{1}{i \hbar}\left[\frac{\hbar^{2}}{2 m} \nabla^{2}+V\right] .
$$

The spatiotemporal histories of the PO $\chi=\chi(t)$ evolve according to $\frac{d \chi}{d t}=u$, determined by $\eta$, which in turn evolves according to the equation $\frac{\delta \eta}{\delta t}=F$.

In a quantum theory in which the PO evolves according to a stochastic law, the generalization is less evident but it is still there. The law $u$ for the PO $\chi$ amounts to the

${ }^{15}$ See [Allori 2013a; 2013b], [Allori 2015a; 2015b; forthcoming]. 
specification of the possible probability distributions, for example, providing the generator or the transition probability of a Markov process. For example, in GRWm the PO is the mass density field, $\chi=m^{\psi}(r, t)$, and the $u$ function is given by its definition in terms of the nomological variable $\eta=\psi$. The $\eta$ variable evolves according to the function $F$, which the stochastic GRW-evolution expressed in terms of $\chi$. Correspondingly, the spatiotemporal histories of the PO evolve according to $\frac{d \chi}{d t}=u$.

Let us now consider the case of quantum theories with a PO in space-time, like GRWf, in which $\chi$ is the set of flashes $\chi=\left\{\left(X_{1}, T_{1}\right), \ldots,\left(X_{k}, T_{k}\right), \ldots\right\}^{\psi}$. Here there is no $u$ function because the theory is presented directly in a space-time framework. Nonetheless, the possible histories of the $\mathrm{PO}$ are determined by the equation for the distribution of flashes in terms of the wave function. As in GRWm, the nomological variable $\eta$ is the wave function, which evolves to the stochastic GRW-evolution.

Before moving to the emergence of the classical world in the PO framework, let me make some final remarks. Notice that any choice of $\chi, \eta, u$ and $F$ will never be logically forced or determined by experiments. Theories are in this sense underdetermined by data: once the PO $\chi$ is fixed, different mathematical choices of $\eta$, the variables to implement the law of motion of $\chi$, can be made, since the same trajectories can be generated by different $\eta$. For instance, in Newtonian mechanics, the same particles trajectories are generated by a potential $V$ and the potential $V=V^{\prime}+$ constant, given that the force will be the same. Similarly, as already mentioned and as it has been shown in [AGTZ 2008], Bohmian mechanics as a theory of particles can be reformulated in terms of a GRW-evolving wave function, and that GRW as a theory of flashes can be reformulated in terms of a linearly evolving wave function. These theories are 'physically equivalent:' as long as the spatiotemporal histories of the $\mathrm{PO}$ are the same, the details of the evolution of the nomological variable do not matter. Thus, we have different way of freely choose our theories and their components (that of course must be constrained by the comparison with the experimental data):

- Freedom in choosing the PO $\chi$ : changing $\chi$ we change the theory, since we are changing the mathematical objects representing matter. This is what happens for example in theories like string theory, in which the basic objects are onedimensional, rather than dimension-less, as in classical mechanics.

- Freedom in choosing $\eta$ : changing $\eta$ does not amount to a change in the theory if the evolution of the PO stays the same. This is what happens, for example, in classical mechanics using two potentials that differ by a constant. If instead we change $\eta$ such that the evolution for $\chi$ changes, then we have a different theory. This is what happens between Bohmian mechanics and GRWp3.

- Freedom in choosing $u$ : changing $u$ amounts to change the law of evolution for the $\mathrm{PO}$, so the theory may well change. An example of a change of $u$ keeping fixed the $\mathrm{PO}$ is given by the passage from classical to Bohmian mechanics. 
- Freedom in choosing $F$ : changing $F$ we change the law of evolution for $\eta$ and two things can happen. Either this change also affects the evolution of the PO or it does not. In the former case, the theory changes, as we can see if we compare classical mechanics with classical electrodynamics, in which the change is $F$ amounts to the change in the potential (we add the electromagnetic potential to the gravitational one). In the latter case, the theory is the same as before. The same $u$ for a given $\chi$ means that the theories give rise to the same trajectories for the PO even if we have a different $F$ : they are physically equivalent. An example of this is GRWf and its linear reformulation.

\section{The Emergence of the Classical World}

In this section I am going to show that within the PO approach one can set up a framework to situate the emergence of the classical world from quantum theories, based on the fact that the general scheme of mechanics is the same in quantum and classical theories ${ }^{16}$.

How does the classical world emerge from the description provided by quantum mechanics? Usually, the classical limit is associated with the limit $\hbar \rightarrow 0$, meaning by this $\hbar \ll A_{0}$, where $A_{0}$ is some characteristic action of the corresponding classical motion [Maslov \& Fedoriuk 1981]. This condition is often regarded as equivalent to another which involves some relevant length scales of the motion: $\lambda_{d B} \ll$ $L$, where $\lambda_{d B}$ is the de Broglie wavelength of the 'particle' and $L$ is the scale on which the potential varies given by $L=\sqrt{\left|\frac{V^{\prime}}{V^{\prime \prime \prime}}\right|}$, where $V^{\prime}$ and $V^{\prime \prime \prime}$ denote respectively the first and the third derivative of the potential ${ }^{17}$. These two length scales allow to define the classical limit in terms of parameter $\varepsilon=\frac{\lambda_{d B}}{L}$. The basic idea is that the classical world emerges whenever $\varepsilon \rightarrow 0$. What exactly this means depends on our view on quantum theories. Within the framework of standard quantum mechanics, the classical limit is often discussed in terms of the Ehrenfest theorem: if the initial wave function is a narrow wave packet, the packet moves approximately according to Newton's second law. Thus, the emergence of the classical world is associated with the formation and preservation of narrow wave packets. While any wave packet will typically spread and consequently the classical approximation will break down, decoherence, namely the interaction of the system with its environment, helps in keeping the packet narrow

\footnotetext{
${ }^{16}$ Notice that this approach is strictly bottom-up: the basic idea is that we can explain why macroscopic objects behave classically assuming that they composed by microscopic entities governed by quantum theory. In contrast, approaches like the ones developed in [Fortin \& Lombardi, this volume], and [Arsenijevic, Jeknic-Dugic, Dugic, this volume] are explicitly top-down.

17 See [Allori 2007], [Allori \& Zanghi 2009], [ADGZ 2001].
} 
[GJKKSZ 1996] ${ }^{18}$. In the PO framework, instead, we have more and different resources. In fact, the wave function is not the only ingredient of the theory. Indeed, it is not even the main ingredient: matter is described by the $\mathrm{PO}$, while the wave function is a nomological variable, needed to implement the PO's temporal evolution. The main idea is therefore that that classical behavior arises when the trajectories of the PO generated by the quantum theory approximate the classical trajectories as the relevant parameter $\varepsilon$ goes to zero. Pictorially, this is equivalent to say that there is a macroscopic scale, defined by $\lambda_{d B}$ and $L$, on which trajectories 'look' classical. Consider a very big poster, like the ones one encounters in the walls of the subway: if one looks closely, they are made of small colored circles, but when one looks from a distance, the single circles blend together to form a face. Similarly, looking at quantum trajectories 'from far away' (i.e. on the macroscopic scale defined by $\rightarrow 0$ ), all the quantum features fade out and the trajectories appear classical. First, I will discuss what this means the case of quantum theories with a $\mathrm{PO}$ of particles, given that the quantum $\mathrm{PO}$ is the same as the classical PO. Then, I will show how one can generalize this to the cases in which the quantum theory has a PO that is different from particles.

As we have seen, the paradigmatic example of a quantum theory of particles is given by Bohmian mechanics. To show the convergence of the quantum trajectories to the classical ones is not an easy task, for $\lambda_{d B}$ is in general a function of the wave function, and $L$ depends on the potential. Thus, the dimensionless parameter $\varepsilon$ may depend in general on a combination of various quantities $x, y, z \ldots$, which relate to the potential and to the wave function. Therefore the limit $\varepsilon(x, y, z, \ldots) \rightarrow 0$ can be taken along different paths (different sequences) in the $x, y, z \ldots$ space. It turns out that the limit $\hbar \rightarrow 0$ is only a special case of the limit $\varepsilon(x, y, z, \ldots) \rightarrow 0$ : it is only one special sequence and it is not able to cover the complexity of the classical world as a whole. Nonetheless, the convergence of quantum trajectories to classical ones, as the parameter $\varepsilon$ goes to zero, can be explicitly derived for special $\operatorname{cases}^{19}$. One such case is when we consider a fixed external potential acting on a particle whose motion is governed by a quasi-classical wave function, namely a wave function of the short wave form, which corresponds to a small de Broglie wavelength $\lambda_{d B}$ when compared to $L$, the length of variation of the potential. Therefore, $\varepsilon$ goes to zero as $\lambda_{d B}$ goes to zero while $L$ is fixed. Another special limit is the one of slowly varying potentials: they are potentials that vary on a scale $L$ which is much greater than the de Brolgie wavelegth. Here, $\varepsilon$ goes to zero as $L$ goes to infinity, and $\lambda_{d B}$ is fixed. It has been shown that in both these cases the quantum trajectories approximate the classical ones in the limit $\varepsilon \rightarrow 0$. Also, in both cases there is the formation of a 'local plane wave:' a wave function that locally can be

${ }_{18}$ For more on decoherence and its role on the classical limit of quantum mechanics, see [Fortin \& Lombardi, this volume].

${ }^{19}$ See [Allori 2001; 2007], [Allori \& Zanghi 2009]. 
regarded as a plane wave having a local wave length. That is, a local plane wave is a wave function which is slowly varying over a distance of the order of the de Broglie wavelength. The idea is that classical behavior arises when a local plane wave forms. Such local plane wave can be thought of a sum of non- interacting 'virtual' wave packets $\psi_{\mathrm{k}}$, each with an almost constant wave length $\lambda_{d B} k$ as shown in figure 1 . Analysis shows that this local plane wave structure is preserved by the dynamics: one the local plane wave is formed, in a relevant sense it keeps guiding the particle's motion classically. In fact, not all the wave function is relevant to determine the motion: there is an effective guiding wave packet which moves the particles along a classical trajectory. This effective wave packet is the part of the wave function in a local neighborhood of the trajectory at any given time. As it has been shown in [Allori 2001;2007], for the model considered, the classical behavior is not preserved for long times due to interference terms coming from the different components of the local plane waves. In a more realistic model, the environmental degrees of freedom (namely decoherence) would take care of suppressing the superpositions of spatially separated wave functions ${ }^{20}$.

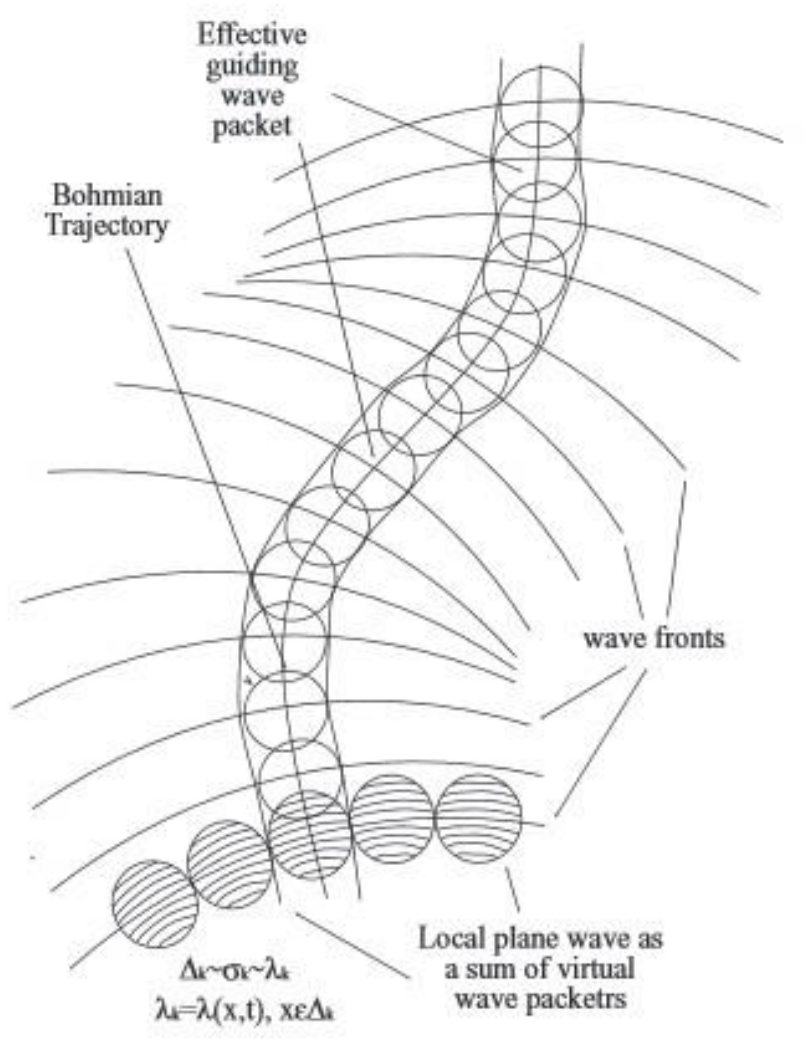

Figure 1: Local plane wave

${ }^{20}$ This result has also been obtained by [Holland, this volume]. 
Now, let us consider the case of quantum theories whose PO is different from the one of classical mechanics. For example, consider GRWm, in which the PO is a matter density three-dimensional field. What does it mean that quantum trajectories converge to classical ones in this framework? There is practically no literature on the subject but presumably what is necessary is that there is a macroscopic scale in which: (1) the quantum PO converges to the classical PO; and (2) the quantum trajectories of the PO converge to the classical ones. The first points should be understood as saying that, on the relevant scale, certain quantum microscopic details about matter are obscured and irrelevant, and matter appears as to be made by something else: even if it is not fundamentally made of particles, it looks like it is. A sphere from far away looks like a one-dimensional point, and a hose from far away looks like a one-dimensional string. Similarly, the continuous three-dimensional matter field which constitute the PO of theories like GRWm from far away would look as if matter is concentrated only in its peaks, even if actually there is matter everywhere. The second condition, namely that the quantum trajectories of the PO, the matter density, would converge to the classical one, is in certain respects similar to the case of Bohmian mechanics: when focusing on the peaks of the matter field, their trajectories will converge to classical trajectories. But the situation is a little more complicated in GRWm because the matter density field has superpositions, generated by the superposition of the wave function. As we saw, GRWm gets rid of them as the wave function spontaneously collapses into one of the terms of the superposition with a rate that depends on $N$, the number of 'particles' in the system. That is, the more macroscopic the body, the quicker its mass density will collapse into one of the terms of the superpositions. Therefore, in order for the classical behavior to emerge, first one needs the wave function to collapse, so that the matter density behaves accordingly: this presumably happens on a time scale that is inversely proportional to the rate of collapse, which is proportional to the number of 'particles' in the system. Then, the remaining term in the matter field has to be 'looked at' on a scale in which it seems particle-like: this presumably happens on a spatial scale of the order of $\sigma$, the width of the Gaussian by which the wave function is multiplied during the collapse. The matter density will start spreading, given that the wave function will spread just as in Bohmian mechanics. In this theory, though, the local plane wave would form and be preserved by the dynamics, ensuring the classical behavior. Here, one would need the collapse of the wave function to induce the collapse of the matter density field in order to prevent matter from spreading out. Similar considerations hold true for Sm, with the difference that here one does not need the collapse since every term of the superposition is realized in a different world. No one has proven yet that in the relevant macroscopic scale the classical world emerges in GRWm or Sm, and it is an interesting research project. In particular, it would interesting to see the role of local plane waves and their interplay with the wave function collapse, as well as the role of decoherence. Details aside, it seems perfectly clear how to formulate the question of the 
classical limit in the framework of quantum theories with a PO, even if they are not particle theories: both here and in the case of Bohmian mechanics in order to obtain classical behavior one has to focus on the trajectories of the PO, not on the wave function, and one has to find the conditions under which these trajectories approximate the classical ones.

Finally, what about theories directly formulated in space-time, like GRWf or Sf? The idea is that the classical world would emerge when the quantum distribution of flashes in space-time approximate the space-time classical trajectories of particles. Presumably, there is a macroscopic scale in which the set of flashes 'clump together' in space-time to form classical patterns. In GRWf, the flashes are the collapse centers of the wave function, so the more massive (i.e. classical) the object is, the more flashes there will be. There is no literature that explores the relevant macroscopic scale for the emergence of classical behavior in this context, and what mechanics or structures this emergence involves. Nonetheless, given the parameter in GRWf, if the number $N$ of the degrees of freedom in the wave function is large, as in the case of a macroscopic object, the number of flashes is also large, of the order of $10^{8}$ flashes per second. Therefore, it seems reasonable to maintain that large numbers of flashes can form macroscopic shapes, such as tables and chairs.

Notice that in standard quantum mechanics the emergence of classicality is always connected to the permanence of a narrow wave function during the motion. Instead in Bohmian mechanics it is exactly the opposite. In fact, the crucial feature of the classical limit in Bohmian mechanics is the formation of very spread out wave function, namely the local plane wave. Thus, only in Bohmian mechanics we can explain the emergence of the classical behavior for delocalized wave functions. In the case of GRWm, classicality arises as long as the matter density field is concentrated and evolves along a classical path, and this is seemingly ensured by the wave function collapse.

Even if there is still work to be done, the PO has an advantage over the competitor wave function ontology approach as proposed by [Albert 1996], and then developed by [Lewis 2005] and [Ney 2012]. In fact, as we just saw, in the PO approach the classical limit of quantum mechanics is understood as the limit in which the spatiotemporal trajectories of the $\mathrm{PO}$ in the quantum theory converge to the classical trajectories. The difficulties one finds in this framework are merely technical, not conceptual: the route to derive the classical behavior from quantum theories is clear. In contrast, the wave function ontology approach considers quantum theories fundamentally as theories in which matter is made of the wave function. Given that, as we have previously mentioned, the wave function is not an object that is defined one three-dimensional space, it is unclear how to define the classical limit. This is obviously not an argument that the classical limit in this approach cannot be defined: to say that the road is steep does not imply it is necessarily impossible to travel. Nonetheless, it is 
unclear why one would want to go home struggling uphill when there is a nice route that brings us home downhill.

Similar arguments can be raised against the various top-down approaches to the classical limit: in the PO approach, like any other bottom-up approach, macroscopic properties are recovered in terms of their microscopic quantum constituents in a way that looks very similar to the derivation of these properties in terms of their microscopic classical constituents. That is, in the framework of classical mechanics, which postulated that everything is made of microscopic point-like particles, the solidity of a table or the transparency of a window are explained (exclusively) in terms of these microscopic particles and their mutual interactions. In the PO approach to quantum theories, we can do what is usually claimed that cannot be done. Namely, that one can derive the properties of macroscopic objects in terms of their microscopic constituents. The topdown approaches, instead decide to recover these properties differently, in a way that is still to be uncontroversially identified. As in the case against the wave function ontology approach, there is a question of motivation: why take the less travelled road if it is not necessary? What is the advantage? We are scientific realists: we do not need to assert our independence of thought, or to show our ability to overcome difficulties, or the like. What we need to do is explain the world around us in terms of our best fundamental physical theories. And more often than not the best explanation is the less convoluted and most boring alternative.

\section{Conclusion}

In the PO framework, a satisfactory fundamental physical theory represents matter using a three-dimensional entity defined on a microscopic (fundamental) scale. Given this, there is more of continuity between quantum and classical theories than one wuld have imagined in the first place. As it has been argued in [Allori 2015b], there is no need to invoke a paradigm shift, or a quantum revolution in order to explain the behavior of macroscopic objects in terms of quantum mechanics.

The three-dimensionality of the PO guarantees that we do not need to worry about the emergence of a three-dimensional space from something of higher dimensions. In addition, a microscopic PO that constitutes the building blocks of everything else is able to ground the classical limit as we have shown in the previous section: classical (macroscopic) objects are composed of quantum (microscopic) PO, and their classical behavior emerges from quantum reality on suitable macroscopic scales which are defined in terms of the evolution of the quantum PO. Even if the problem of the classical limit has been proven difficult to be rigorously treated within the PO framework, in contrast with the alternative approaches the challenges are not a matter of conceptualization. Heuristics and conjectural arguments can be provided to explain the emergence of classical behavior from classical theories, and more research just needs to done to improve them. 


\section{References}

- [Albert 1996] Albert, D.Z.: Elementary Quantum Metaphysics. In: J. Cushing, A. Fine, and S. Goldstein (eds.), Bohmian Mechanics and Quantum Theory: An Appraisal: 277-284. Kluwer (1996).

- [Allori 2001] Allori, V.: Decoherence and the Classical Limit of Quantum Mechanics. Ph. D. Thesis, Department of Physics, University of Genova, Italy (2001).

- [Allori 2007] Allori, V.: Fundamental Physical Theories: Mathematical Structures Grounded on a Primitive Ontology. Ph. D. thesis, Department of Philosophy, Rutgers University (2007).

- [Allori 2013a] Allori, V.: On the Metaphysics of Quantum Mechanics. In: S. Lebihan (ed.),Precis de la Philosophie de la Physique. Vuibert (2013).

- [Allori 2013b] Allori, V.: Primitive Ontology and the Structure of Fundamental Physical Theories. In: D. Z. Albert, A. Ney (eds.), The Wave Function: Essays in the Metaphysics of Quantum Mechanics: 58-75. Oxford University Press (2013).

- [Allori 2015a] Allori, V.: Primitive Ontology in a Nutshell. International Journal of Quantum Foundations 1 (3): 107-122 (2015).

- [Allori 2015b] Allori, V.: Quantum Mechanics and Paradigm Shift. Topoi 32 (2): 313-323 (2015).

- [Allori 2015 c] Allori, V.: Maxwell's Paradox: Classical Electrodynamics and its Time Reversal Invariance. Analytica 1: 1-19 (2015).

- [Allori manuscript] Allori, V.: Primitive Ontology and Scientific Realism.

- [Allori forthcoming] Allori, V.: How to Make Sense of Quantum Mechanics (and More): Fundamental Physical Theories and Primitive Ontology. In: U. Edvinsson (ed.), The Mammoth Book of Quantum Mechanics Interpretations. Open Academic Press (forthcoming).

- $\quad$ [AGTZ 2008] Allori, V., S. Goldstein, R. Tumulka, N. Zanghi: On the Common Structure of Bohmian Mechanics and the Ghirardi-Rimini-Weber Theory. The British Journal for the Philosophy of Science 59 (3): 353-389 (2008).

- [AGTZ 2011] Allori, V., S. Goldstein, R. Tumulka, N. Zanghi: Many-Worlds and Schrödinger's First Quantum Theory. The British Journal for the Philosophy of Science 62 (1), 1-27 (2011).

- $\quad$ [AGTZ 2014] Allori, V., S. Goldstein, R. Tumulka, N. Zanghi: Predictions and Primitive Ontology in Quantum Foundations: A Study of Examples. The British Journal for the Philosophy of Science 65 (2): 323-352 (2014).

- [Allori \& Zanghi 2009] Allori, V., N. Zanghi: On the Classical Limit of Quantum Mechanics. Foundations of Physics 39 (1): 20-32 (2009).

- [Allori \& Zanghi 2004] Allori, V., N. Zanghi: What is Bohmian Mechanics. International Journal of Theoretical Physics 43: 1743-1755 (2004). 
- [Arsenijevic, Jeknic-Dugic, Dugic, this volume] Arsenijevic, M., J. Jeknic-Dugic, M. Dugic: A Top-down versus a Bottom-up Hidden-variables Description of the SternGerlach Experiment. This volume.

- [Bai \& Stachel, this volume] Bai, T., Stachel, J.: Bohr's Diaphragms. This volume.

- [Baumgarten, this volume] Baumgarten, C.: Minkowski Spacetime and QED from Ontology of Time. This volume.

- [Bell 1987] Bell, J.S.: Speakable and Unspeakable in Quantum Mechanics. Cambridge University Press (1987).

- [BGG 1995] Benatti, F., G.C. Ghirardi, R. Grassi, R.: Describing the Macroscopic World: Closing the Circle within the Dynamical Reduction Program. Foundations of Physics 25: 5-38 (1995).

- [Bohm 1952] Bohm, D.: A Suggested Interpretation of the Quantum Theory in Terms of "Hidden" Variables, I and II. Physical Review 85: 166-193 (1952).

- [Chiatti and Licata, this volume] Chiatti, L., Licata, I.: Fluidodynamical Representation and Quantum Jumps. This volume.

- [Esfeld forthcoming] Esfeld, M.: How to account for Quantum non-locality: Ontic Structural Realism and the Primitive Ontology of Quantum Physics. Synthese (forthcoming).

- [Everett 1957] Everett, H.: Relative State Formulation of Quantum Mechanics. Review of Modern Physics 29: 454-462 (1957).

- [Fortin \& Lombardi, this volume] Fortin, S. Lombardi, O.: A Top-down View of the Classical Limit of Quantum Mechanics. This volume.

- [GRW 1986] Ghirardi, G.C., A. Rimini, T. Weber: Unified Dynamics for Microscopic and Macroscopic Systems. Physical Review D34: 470-491 (1986).

- [GJKKSZ 1996] Giulini, D.,E. Joos,C. Kiefer, G.C. Kumpsch, I. Stamatescu, H.D. Zeh: Decoherence and the Appearance of a Classical World in Quantum Theory. Springer-Verlag, Berlin (1996).

- [Holland, this volume] Holland, P.: The Quantum State as Spatial Displacement. This volume.

- [Kitcher 1993] Kitcher, P.: The Advancement of Science: Science Without Legend, Objectivity without Illusions, Oxford University Press (1993).

- [Kastner, this volume] Kastner, R.: Beyond Complementarity. This volume.

- [Lewis 2005] Lewis, P.: Interpreting Spontaneous Collapse Theories. Studies in History and Philosophy of Modern Physics 36: 165-180 (2005).

- [Maslov \& Fedoriuk 1981] Maslov, V.P., M. V. Fedoriuk: Semi-Classical Approximation in Quantum Mechanics, D. Reidel Publ. Co., Dordrecht, Holland (1981).

- [Monton 2006] Monton, B.: Quantum Mechanics and 3N-Dimensional Space. Philosophy of Science 73 (5): 778-789 (2006). 
- [Ney 2012] Ney, A.: The Status of our Ordinary Three Dimensions in a Quantum Universe. Nous 46(3), 525-560 (2012).

- [Tumulka 2006a] Tumulka, R.: A Relativistic Version of the Ghirardi-Rimini-Weber Model. Journal of Statistical Physics 125: 821-840 (2006).

- [Wallace 2002] Wallace, D.: Worlds in the Everett Interpretation. Studies in the History and Philosophy of Modern Physics 33: 637-661 (2002). 